Proceedings of the II Symposium on Positron Emission Tomography, Kraków, September 21-24, 2014

\title{
Time-of-Flight Positron Emission Tomography with Resistive Plate Chamber Detectors: An Unlikely but Promising Approach
}

\author{
M. Couceiro ${ }^{a, b, *}$, P. $\operatorname{Crespo}^{a, c}$, A. Blanco ${ }^{a}$, N.C. Ferreira $^{d, e}$, L. Mendes $^{d, e}$, \\ R. FERreira MARQues ${ }^{a, c}$ And P. Fonte ${ }^{a, b}$ \\ ${ }^{a}$ Laboratório de Instrumentação e Física Experimental de Partículas, Departamento de Física, \\ Universidade de Coimbra, Rua Larga, 3004-516 Coimbra, Portugal \\ ${ }^{b}$ Instituto Politécnico de Coimbra, ISEC, Rua Pedro Nunes, Quinta da Nora, 3030-199 Coimbra, Portugal \\ ${ }^{c}$ Departamento de Física, Faculdade de Ciências e Tecnologia, Universidade de Coimbra, \\ Rua Larga, 3004-516 Coimbra, Portugal \\ ${ }^{d}$ Instituto Biomédico de Investigação da Luz e Imagem, Faculdade de Medicina, Universidade de Coimbra, \\ Azinhaga Santa Comba, Celas, 3004-516 Coimbra, Portugal \\ ${ }^{e}$ Instituto de Ciências Nucleares Aplicadas à Saúde, Faculdade de Medicina, Universidade de Coimbra, \\ Azinhaga Santa Comba, Celas, 3004-516 Coimbra, Portugal \\ The cost-effectiveness of resistive plate chamber detectors and their very good timing characteristics, open the \\ possibility to build affordable time-of-flight positron emission tomography systems with a large axial field-of-view. \\ Simulations suggest that, under reasonable assumptions, the absolute 3D true sensitivity, spatial resolution, and \\ noise equivalent count rate of such systems for human whole-body screening, may exceed that of present crystal- \\ based PET technology. However, due to the lack of energy resolution, although having energy sensitivity, the \\ scatter fraction is expected to be considerably higher than that presented by crystal-based PET scanners. In the \\ present paper, the simulation work done so far to access the expected performance of a resistive plate chamber \\ time-of-flight-PET system with $2400 \mathrm{~mm}$ length axial field-of-view, a time resolution of $300 \mathrm{ps}$ full width at half \\ maximum for photons pairs, and depth-of-interaction information, will be revised.
}

DOI: 10.12693/APhysPolA.127.1453

PACS: 87.57.uk, 29.40.Cs, 87.57.cf, 87.57.cm

\section{Introduction}

The resistive plate chamber (RPC) time-of-flight (TOF) positron emission tomography (PET) concept [1] is based on the converter plate principle [2], depicted in Fig. 1.

Simple and economic construction, even for large area covering, good timing resolution of $300 \mathrm{ps}$ full width at half maximum (FWHM) for $511 \mathrm{keV}$ photon pairs [1], excellent position accuracy of $38 \mu \mathrm{m}$ [3], and the capability to resolve the depth-of-interaction (DOI) [4], make RPC detectors an alternative for developing PET scanners with a large axial field-of-view (AFOV) almost free of parallax errors and with TOF capability. As the major disadvantage when compared to inorganic crystal scintillation detectors, one can point out the lack of energy resolution. However, for the detection of photons, RPCs present an energy sensitivity [5, 6], which, for PET applications, is equivalent to an energy discrimination threshold of $300 \mathrm{keV}$ in terms of rejection of coincidences involving scattered photons [1].

* corresponding author; e-mail: couceiro@coimbra.lip.pt

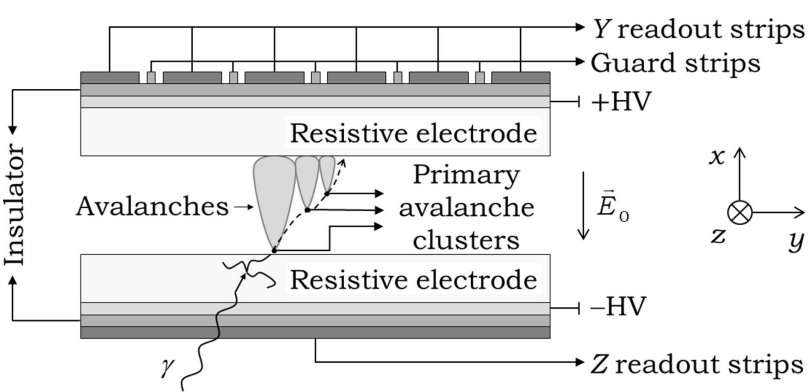

Fig. 1. Scheme of a single-gap timing RPC detector with position readout, depicting the converter plate principle.

In the present paper, the simulation work done so far to access the expected performance of a full-body length RPC TOF-PET scanner, will be briefly revised. Full description of (and details on) the simulation work done so far can be found in Refs. [6-11].

\section{Simulation setup and procedures}

To access the expected performance of a large AFOV RPC TOF-PET scanner, in what concerns the sensitivity, spatial resolution (SR), scatter fraction (SF), count rates (CR), and noise equivalent count rate (NECR), 
as measured by the National Electrical Manufacturers Association (NEMA) NU2 standards [12, 13], simulations were performed with programs developed with the GEANT4 toolkit $[14,15]$. For the sensitivity test, the 1994 and the 2001 versions of the NEMA NU2 standards [12, 13] were used, while for the SR, SF and NECR tests, only the NEMA NU2-2001 standards [13] were employed.

In what follows, the general setup employed for the simulations concerning each of the aforementioned performance parameters will be given shortly.

\subsection{Sensitivity}

For the sensitivity test following the NEMA NU2-1994 standards [12], the scanner was modelled as a right circular annulus with $927 \mathrm{~mm}$ inner diameter and an AFOV ranging from $150 \mathrm{~mm}$ to $2400 \mathrm{~mm}[6,7]$. The test phantom consisted in a $1750 \mathrm{~mm}$ axially extended version $[6,7]$ of the phantom defined in the NEMA NU2-1994 standards [12]: a $3 \pm 1 \mathrm{~mm}$ thick right circular cylindrical polymethyl methacrylate (PMMA) shell, with an outer diameter of $203 \pm 3 \mathrm{~mm}$ and an inner axial length of $190 \pm 1 \mathrm{~mm}$, with the core filled with a given activity diluted in water. The physics list employed was entirely based on the standard electromagnetic physics (SEP) models of the GEANT4 toolkit $[14,15]$, and the source was defined as positrons at rest, uniformly distributed in the core of the test phantom $[6,7]$.

For the sensitivity test following the NEMA NU2-2001 standards [13], the scanner was defined in a approxilar way, but with a diameter of $920 \mathrm{~mm}$ and a fixed AFOV of $2400 \mathrm{~mm}$ [9], and the test phantom consisted in that defined in the NEMA NU2-2001 standards [13]: a line source with an axial length of $700 \mathrm{~mm}$, which is surrounded by a variable thickness of aluminium by means of five concentric $2.5 \mathrm{~mm}$ thick cylindrical sleeves that are added in turn around the line source. An axially extended version of the phantom, with a length of $2400 \mathrm{~mm}$, was also simulated [9]. The physics list employed was based on the low-energy electromagnetic package (LEP) of the GEANT4 toolkit $[14,15]$, and the source consisted in back-to-back photons uniformly distributed in the core of the line source [9].

In both cases, the phantoms were centred in the scanner field-of-view (FOV), photons were fully tracked in the phantom, and the points were the photons impinged the inner surface of the detection annulus were taken as the detection points $[6,7,9]$.

\subsection{Scatter fraction, count rates and noise equivalent count rate}

For the SF, CR, and NECR tests, the scanner was defined as depicted in Fig. 2: a hollow parallelepiped with four detections heads, each containing a stack of twenty double-module multi-gap RPCs, each module having six $200 \mu \mathrm{m}$ thick glass plates separated by five $350 \mu \mathrm{m}$ thick amplification gaps, each module having its own axial readout electrode, the transaxial electrode being shared by both modules $[10,11]$.

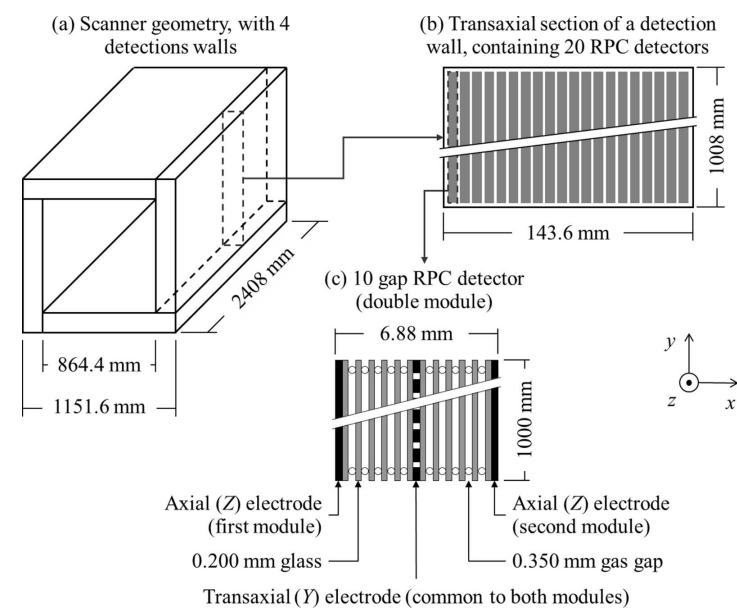

Fig. 2. Sketch of the RPC TOF-PET system [10, 11]: (a) scanner, consisting of 4 detection walls; (b) detection wall containing a stack of 20 double-module multi-gap RPC detectors; (c) RPC detector with two detection modules, each containing 6 glass plates with thicknesses of $200 \mu \mathrm{m}$, separated by 5 amplification gaps with individual thicknesses of $350 \mu \mathrm{m}$, with a common transaxial and two independent axial readout electrodes.

The test phantom, which was centred in the FOV, consisted in that defined in the NEMA NU2-2001 standards [13]: a right circular cylinder of polyethylene with specific gravity of $0.96 \pm 0.01$, an axial length of $700 \pm 5 \mathrm{~mm}$, and a diameter of $203 \pm 3 \mathrm{~mm}$, in which a hole with $6.4 \pm 0.2 \mathrm{~mm}$ diameter is drilled parallel to the central axis of the phantom but displaced $45 \pm 1 \mathrm{~mm}$ from it, which serves to accommodate a line source consisting in a right circular cylindrical annulus (also of polyethylene) with inside and outside diameters of, respectively, $3.2 \pm 0.2 \mathrm{~mm}$ and $4.8 \pm 0.2 \mathrm{~mm}$, the core being filled with a given activity diluted in water. An axially extended version of the phantom, with an axial length of $1800 \mathrm{~mm}$, was also simulated [10, 11]. For both phantoms, the source consisted in ${ }^{18} \mathrm{~F}$ nuclei uniformly distributed in the core of the line source $[10,11]$.

The physics list employed was based on the SEP models of the GEANT4 toolkit $[14,15]$, except for $[10,11]$ : (1) the Rayleigh interaction, for which the LEP provided by the GEANT4 toolkit $[14,15]$ was used, and (2) the positron annihilation physics, for which the routine provided by GATE [16], accounting for the photon acollinearity [16], was implemented in the physics list of the programs developed with the GEANT4 toolkit $[14,15]$. Photons were fully tracked in the phantom and in the scanner, and electrons were fully tracked if generated inside a glass plate, the track being killed if they reached an amplification gap $[10,11]$.

\subsection{Spatial resolution}

For the SR test, the scanner was defined as mentioned in Sect. 2.2, except for the detectors, which were set to 40 single-module multi-gap RPC detectors, approxilar to those presented in Fig. 2, each having its own transaxial 
and axial readout electrodes $[8,11]$. The phantom consisted in a spherical point-like water core with $1 \mu \mathrm{m}$ diameter enclosed in a $2 \mathrm{~mm}$ outer diameter shell of PMMA, placed in the scanner center and in the central transaxial plane but displaced $100 \mathrm{~mm}$ in both the scanner $X$ and $Y$ directions $[8,11]$. The physics list employed in the SR test, was approxilar to that employed in the SF, $\mathrm{CR}$ and NECR tests, and the particle tracking was performed in the same way $[8,11]$. The source consisted of positrons at rest uniformly distributed in the phantom core $[8,11]$.

\section{Simulation data processing}

The processing of simulation produced data was performed in cascade stages by programs developed externally to the programs developed with the GEANT4 toolkit $[14,15]$. In what follows, those stages will be addressed.

\subsection{Decay times}

For all tests, decay times randomly drawn from an exponential distribution with a given activity and the ${ }^{18} \mathrm{~F}$ decay constant (and $\beta^{+}$branching ratio, if the source consisted in positrons at rest or in back-to-back photons), were assigned to the photon flight times of single hits, which were then affected by a $90 \mathrm{ps} \sigma$ Gaussian time jitter to account for the $300 \mathrm{ps}$ FWHM TOF resolution [6-11].

The activity considered for the sensitivity tests (both with the 1994 and the 2001 versions of NEMA NU2 standards $[12,13])$, was set to a value low enough so that losses due to detector dead time and random coincidences were negligible $[6,7,9,11]$. For the $\mathrm{SR}$ test, an activity of $370 \mathrm{kBq}(10 \mu \mathrm{Ci})$ was used [8, 11]. The activity considered for the $\mathrm{SF}$ test was of $37 \mathrm{kBq}(1 \mu \mathrm{Ci})$, and for the $\mathrm{CR}$ and NECR tests, activities ranging from $37 \mathrm{kBq}$ $(1 \mu \mathrm{Ci})$ to $370 \mathrm{MBq}(10 \mathrm{mCi})$ were used $[10,11]$.

\subsection{Detection efficiency}

For the sensitivity test, the detection efficiency was modelled according to the curves presented in Fig. 3, obtained by simulating (with the GEANT4 toolkit $[14,15]$ ) the extraction efficiencies of stacks of 61 and 121 glass plates with individual thicknesses of $0.4 \mathrm{~mm}$, separated by, respectively, 60 and 120 amplification gaps with individual thicknesses of $350 \mu \mathrm{m}[6,9,11]$.

For the SR, SF, CR, and NECR tests, photons were fully tracked in the scanner, the detection efficiency being then accounted for in the simulation.

\subsection{Detector readout}

For the sensitivity tests, the detection points were binned according to the segmentation of the General Electric (GE) Advance scanner, and the detector readout was not accounted for $[6,7,9,11]$.

For the SR, SF, CR, and NECR tests, the detection points of single events were considered to be those on the opposite side of the amplification gap relative to the extraction point, computed along the electron momentum direction $[8,10,11]$. The resulting detection points were

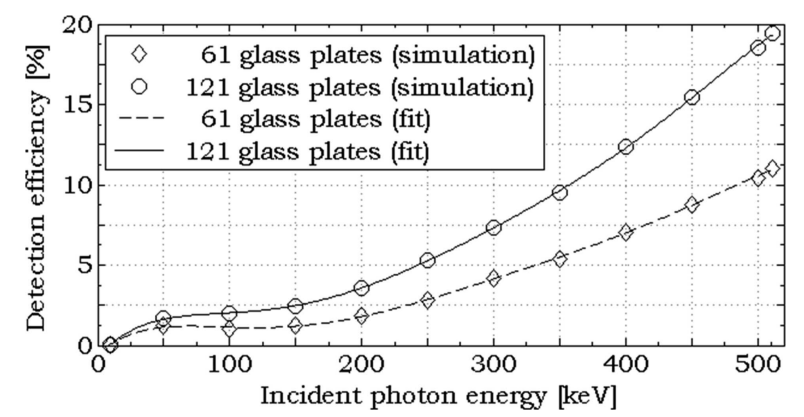

Fig. 3. Detection efficiency of stacks of 61 and 121 glass plates with individual thicknesses of $400 \mu \mathrm{m}$ (defining, respectively, 60 and 120 amplification gaps with individual thicknesses of $350 \mu \mathrm{m}$ ), as a function of the incident photon energy, for photons impinging the first glass perpendicularly to its surface $[6,9,11]$.

then processed to account for the segmentation of the detector as described in Refs. [8, 10, 11], the final detection point being then binned to $2 \mathrm{~mm}$ along the axial and transaxial directions (corresponding to the current pitch of the pickup strips), and to the middle of the detection module, in bins of $3.44 \mathrm{~mm}$ (the thickness of each detection module), leading to a maximum DOI error (along the radial direction) of $1.72 \mathrm{~mm}$ (half the module thickness) $[8,10,11]$. For the SR test, bins of $1 \mathrm{~mm}$ along the transaxial and axial directions, and continuous detection along all directions (transaxial, axial, and radial), were also considered $[8,11]$.

\subsection{Coincidence processing}

Single events resulting from the processing stage to account for the detector readout, were then fed to a coincidence sorter. For the sensitivity test, a $12.5 \mathrm{~ns}$ single time window (STW) coincidence sorter (for which, at a given time, only one coincidence time window, opened by the triggering event, is active), was used, and multiple coincidences were discarded $[6,7,9,11]$. For the SR test, a STW coincidence sorter was also used, but with a 4 ns time window, and multiple coincidences were also discarded [8, 11].

For the SF, CR and NECR tests, 5 ns STW and multiple time window (MTW) coincidence sorters (for which, each event opens its own coincidence time window) were considered, and all possible coincidence pairs (including all those that can be formed from the multiple coincidences) were then processed to account for the performance test $[10,11]$. (For a complete discussion concerning the use of all possible coincidence pairs, refer to Refs. [10, 11].)

\subsection{Geometric acceptance of lines of response}

For the sensitivity test, all coincidences defining lines of response (LORs) that traversed the scanner bore, were retained $[6,7,9,11]$, while for the $\mathrm{SR}$ test, only those LORs that traversed the scanner bore with a polar acceptance angle less than or equal to $9^{\circ}$ were considered $[8,11]$. 
For the SF, CR, and NECR tests, all LORs were tested for several coincidence rejection/acceptance criteria, two of which were $[10,11]$ : (1) LOR intersection with a tight cylinder surrounding the phantom (geometric rejection - GR), and (2) direct TOF reconstructed point falling inside the aforementioned cylinder (geometric TOF rejection - GTOFR).

\section{Results and discussion}

In what follows the results obtained for each performance test will be summarized mainly by means of plots.

\subsection{Sensitivity}

Figure 4 depicts the expected gain in the absolute $3 \mathrm{D}$ true sensitivity of RPC TOF-PET scanners with 60 and 120 amplification gaps along the radial direction, accessed by simulation [6,11], following the NEMA NU21994 standards [12], for the $1750 \mathrm{~mm}$ long phantom and without considering a gain factor due to TOF information $[17,18]$, relative to the experimental value of $1020 \mathrm{kcps} /\left(\mu \mathrm{Ci} \mathrm{cm}^{3}\right)$ reported by Lewellen et al. [19] for the GE Advance scanner with a ring difference of 11 and the standard 190 mm length NEMA NU2-1994 [12] phantom. As it can be seen, and in what concerns the NEMA NU2-1994 [12] absolute 3D true sensitivity, for an RPC TOF-PET scanner to be advantageous over PET systems based on inorganic scintillation crystal detectors, the AFOV must be greater than or equal to $1200 \mathrm{~mm}$, the number of amplification gaps must be at least 120 , and the polar acceptance angle must be greater than or equal to $15^{\circ}$. For an AFOV of $2400 \mathrm{~mm}$ and a polar acceptance angle of $45^{\circ}$, the gain in sensitivity of an RPC TOF-PET scanner with 120 amplification gaps, relative to that reported by Lewellen et al. [19] for the GE Advance scanner with a ring difference of 11 and the strict NEMA NU2-1994 [12] phantom, was of 5.8, having been of 6.2 for full acceptance on the polar angle [11].

Considering a gain factor due to TOF information given by $[17,18]$ :

$$
g_{\mathrm{TOF}}=\frac{2 \phi_{\text {object }}}{c \mathrm{FWHM}_{\Delta t_{\mathrm{TOF}}}}=4.4,
$$

where $\phi_{\text {object }}$ is the diameter of the test phantom $(200 \mathrm{~mm}), c$ is the light speed, and $\mathrm{FWHM}_{\Delta t_{\mathrm{TOF}}}$ is the TOF resolution (300 ps), the maximum gains achieved by a $2400 \mathrm{~mm}$ AFOV RPC TOF-PET scanner with 120 amplification gaps and full acceptance on the polar angle, relative to that reported by Lewellen et al. [19] for the GE Advance scanner with a ring difference of 11 and the strict NEMA NU2-1994 [12] phantom, was found to be of $27.3[6,11]$.

For the sensitivity test following the NEMA NU2-2001 standards [13], the results obtained for an RPC TOFPET scanner with 120 amplification gaps and $2400 \mathrm{~mm}$ length AFOV, without considering a gain factor due to TOF information [17, 18], also showed an expected increased sensitivity relative to PET scanners based on inorganic scintillation crystal detectors [9]. For a value of the polar acceptance angle of $45^{\circ}$, the gain in the absolute

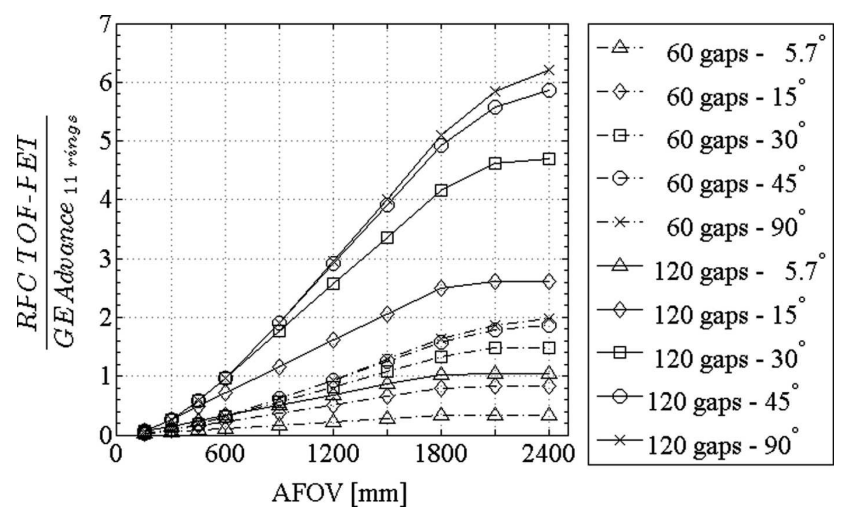

Fig. 4. Expected gain in the absolute 3D True sensitivity of RPC TOF-PET scanners with 60 and 120 amplification gaps [11], following the NEMA NU2-1994 [12] standards, for the $1750 \mathrm{~mm}$ long phantom, relative to that reported by Lewellen et al. [19] for the GE Advance scanner with a ring difference of 11 , for the NEMA NU21994 [12] phantom, without considering a gain factor due to TOF information $[17,18]$ (The polar acceptance angle of $90^{\circ}$ is used to represent full acceptance.).

3D true sensitivity with the $700 \mathrm{~mm}$ length NEMA NU22001 [13] phantom, relative to that reported by Kohlmyer et al. [20] for the GE Advance scanner with the same phantom, was of 4.1 [9]. For the $2400 \mathrm{~mm}$ axially extended phantom, but considering only the $1500 \mathrm{~mm}$ central portion, the gain in absolute $3 \mathrm{D}$ true sensitivity relative to that reported by Kohlmyer et al. [20] for the GE Advance scanner imaging the $700 \mathrm{~mm}$ long phantom, was of 8.4 [9]. Moreover, the results obtained indicate that, for achieving the same planar sensitivity, the time needed to perform a full scan with the RPC TOF-PET scanner is less than that required by commercially available PET scanners based on inorganic scintillation crystals [9].

\subsection{Spatial resolution}

Figure 5 depicts the filtered back projection (FBP) reconstructed image of the central slice obtained by simulation, for the phantom positioned $100 \mathrm{~mm}$ off-axis in both the $X$ and $Y$ directions, along with the point spread function (PSF) and SR (both FWHM and FWTM - full width at tenth maximum) obtained for each of the three Cartesian directions [11]. As it can be seen, the SR of the RPC TOF-PET scanner is expected to be of $\approx 2.3 \mathrm{~mm}$ FWHM and of $\approx 4.9 \mathrm{~mm}$ FWTM, including the detector readout and photon acollinearity effect, but excluding the effect of the positron range $[8,11]$. These values are about twice as better than those reported for current commercially available state-of-the-art PET scanners with TOF information (the Gemini TF from Philips [21], the Discovery 690 from GE [22, 23], and the Biograph mCT from Siemens [24]). Moreover, the effect was found to be strongly dependent on the detector segmentation, but not on the photon acollinearity $[8,11]$. Moreover, the observed effect was much less than the $0.0022 \times D$ (with $D$ the scanner diameter), generally assumed in the literature [25]. 


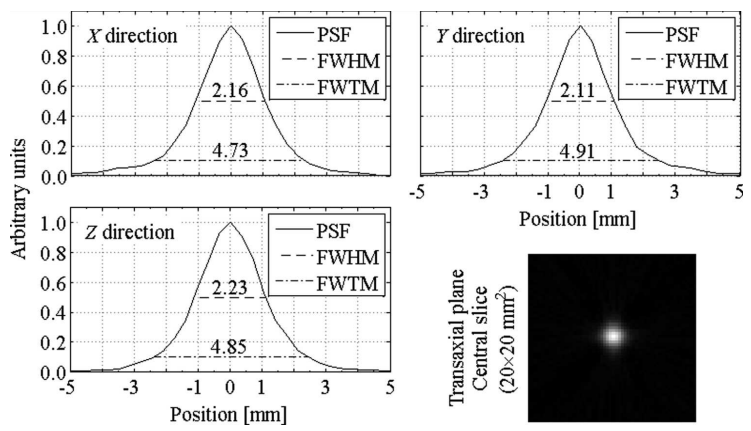

Fig. 5. FBP reconstructed image (bottom right), for the phantom positioned $100 \mathrm{~mm}$ off-axis in both the $X$ and $Y$ directions, along with the PSFs for the three Cartesian directions [11]. The photon acollinearity was accounted for in the simulation, by implementing the annihilation physics provided by GATE [16].

To gain some insight on the effect of photon acollinearity in the SR, coincidence data was obtained mathematically for an ideal cylindrical PET scanner (without considering detector segmentation), with a diameter of $1000 \mathrm{~mm}$ and AFOV of $2400 \mathrm{~mm}$, by randomly drawing annihilation points uniformly distributed in a $100 \mu \mathrm{m}$ diameter spherical volume, and then randomly drawing the photon momentum directions as is performed in the annihilation routine provided by GATE [16], which affects the direction of one of the annihilation photons by a $0.58^{\circ}$ FWHM Gaussian distribution relative to the direction of the other. The times of detection of each generated photon were then computed by approxple light speed kinematics, and were not affected by a time jitter. The direct TOF reconstructed points [26] were then obtained, and the distribution of these distances to the annihilation points, as well as the distances of the LORs to the annihilation points, were computed, both holding a Gaussian distribution with a FWHM of $2.91 \mathrm{~mm}$ [11].

However, the computed distributions are not the PSFs of the reconstructed image acquired in full 3D mode, from which the SR is obtained [13, 27]. These can only be obtained by reconstructing the image and taking the intensity profiles along three orthogonal directions passing through the point of maximum intensity [13, 27], which was performed by creating an image matrix from the direct TOF reconstructed points, as well as by following the complete procedure given in the NEMA NU2-2001 [13] standards, after FBP reconstruction from the $2 \mathrm{D}$ sinograms, with the rebinning of inclined LORs performed by single slice rebinning using TOF information [26], holding FWHM SRs (averaged for the three orthogonal directions) of $97.6 \pm 3.5 \mu \mathrm{m}$ and $103.7 \pm 12.0 \mu \mathrm{m}$ for, respectively, the direct TOF and the FBP reconstructed images [11]. This may indicate an overestimate of the assumed effect of photon acollinearity, and deserves further experimental research with detectors having a high intrinsic spatial resolution, such as those currently employed in the pre-clinical RPC PET prototype [28], which recently reached the final testing stage.

\subsection{Count rates}

The results obtained for the expected CRs for several values of the $\tau_{\mathrm{ps}}$ dead time for position signals, by performing the coincidences with the STW coincidence sorter and both the GR and GTOFR methods for selecting valid coincidences, are presented in Fig. 6 for the true, scatter, and random events.
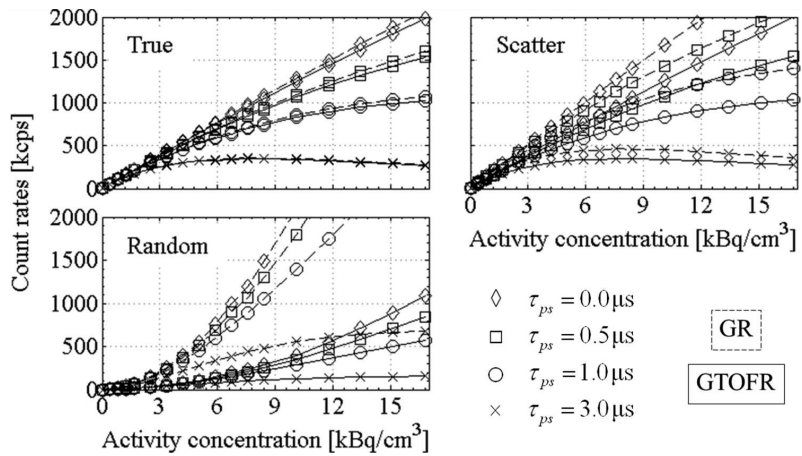

Fig. 6. Expected CRs for the RPC TOF-PET scanner for several values of the $\tau_{\mathrm{ps}}$ dead time for position signals, obtained with the STW coincidence sorter, both GR and GTOFR methods, for the standard $700 \mathrm{~mm}$ length NEMA NU2-2001 [13] phantom [10, 11].

For all values of the $\tau_{\mathrm{ps}}$ dead time for position signals, the GTOFR method was found to marginally decrease the true $\mathrm{CRs}$ from $\approx 1 \%$ to $\approx 5 \%$, relative to those obtained with the GR method, the reduction being higher for higher values of the activity concentration [10, 11]. For the scattered coincidences, the GTOFR was found to reduce the $\mathrm{CRs}$ from $\approx 24 \%$ to $\approx 26 \%$, again with the reduction being more pronounced for higher values of the activity concentration, and independently of the $\tau_{\mathrm{ps}}$ dead time for position signals $[10,11]$. As to the effect of the GTOFR and GR methods on the random CRs, it was found that the former performs excellently in reducing the random $\mathrm{CRs}$ from $\approx 78 \%$ to $\approx 80 \%$ relative to the latter, with the reduction being greater for lower values of the $\tau_{\mathrm{ps}}$ dead time for position signals, and independent of the activity concentration $[10,11]$.

Similar behaviour was found for the MTW coincidence sorter, showing however a systematic increase of all the CRs with the activity concentration, with the increase being greater for the scattered events (from $\approx 10 \%$ to $\approx 30 \%$ ), than for true (up to $\approx 20 \%$ ) and random (up to $\approx 10 \%$ ) coincidences $[10,11]$. These results are depicted in Fig. 7 .

The increased CRs obtained for the scattered coincidences, even for lower values of the activity concentration, indicate that the SF obtained with the STW coincidence sorter is expected to be lower than that obtained with the MTW one. However, the increase in the CRs for the scattered and true coincidences are roughly of the same order of magnitude. Since the NECR benefits from the true CRs by squaring it, worsening only inversely with the scatter and random CRs, it may be expectable that the NECR will be higher for the MTW than for the 


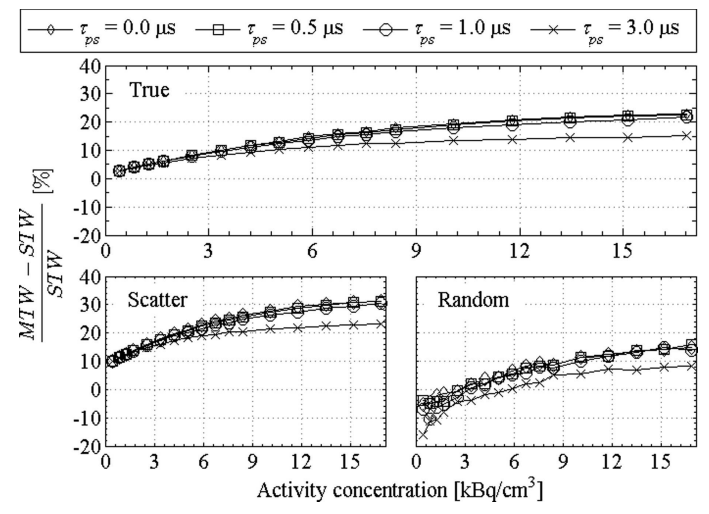

Fig. 7. Percentual deviation, as a function of the activity concentration, for the true, scatter, and random CRs, processed with the MTW coincidence sorter relative to those obtained with the STW coincidence sorter, followed by GTOFR [10, 11] (Simulation data was processed for the standard $700 \mathrm{~mm}$ length NEMA NU22001 [13] phantom.).

STW coincidence sorter. In fact, for the values of the activity concentration present in the phantom with relevance in clinical practice, the MTW coincidence sorter was found to increase the NECR from $\approx 2.5 \%$ (for lower activity concentration) up to $\approx 12.5 \%$ (for higher activity concentrations) relative to that obtained with the STW coincidence sorter $[10,11]$. For this, in what follows only the results obtained with the MTW coincidence sorter will be given.

\subsection{Scatter fraction}

The results obtained for the SF with the coincidences processed with the MTW coincidence sorter and the GTOFR method, are presented in Fig. 8, which also depicts the average SF reported for three commercially available state-of-the-art PET scanners with TOF information (the Gemini TF from Philips [21], the Discovery 690 from GE $[22,23]$, and the Biograph mCT from Siemens [24]). Due to the very low activity in the FOV required for the SF test, for which the probability of pileup to occur is negligible, it was verified that the SF did not depended on the value of the $\tau_{\mathrm{ps}}$ dead time for position signals $[10,11]$.

As it can readily be seen, the SF of the $\mathrm{RPC}$ TOFPET scanner is expected to be of $\approx 52 \%$ for the standard 700 mm long NEMA NU2-2001 [13] phantom, while being of $\approx 54 \%$ for the $1800 \mathrm{~mm}$ axially extended one. This increase in the average SF for the axially extended phantom can be explained by the flatter plateau of the SF profile, and the increased scatter in the phantom along the axial direction.

When compared with the SFs reported for commercially available state-of-the-art PET scanners with TOF information (the Gemini TF from Philips [21], the Discovery 690 from GE $[22,23]$, and the Biograph mCT from Siemens [24]), the SF expected for the RPC TOF-PET scanner is considerably higher. This is due to the lack

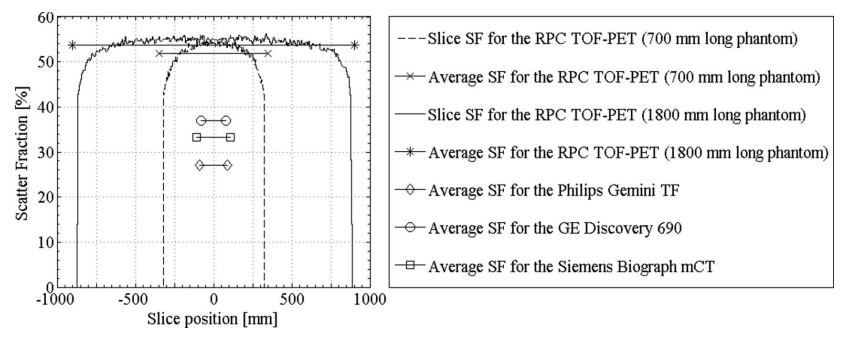

Fig. 8. Expected SF profiles obtained for both the standard $700 \mathrm{~mm}$ long NEMA NU2-2001 [13] and the $1800 \mathrm{~mm}$ axially extended phantoms, along with the corresponding average SFs, for coincidences processed with the MTW coincidence sorter, followed by the GTOFR method [10,11]. Also depicted are the average SFs reported for three state-of-the-art commercially available PET scanners with TOF information (the Gemini TF from Philips [21], the Discovery 690 from GE [22, 23], and the Biograph mCT from Siemens [24]).

of energy resolution of RPC detectors, which can be partially compensated by interposing absorption foils of an appropriate material and with an appropriate thickness in the inner surfaces of the detection heads of the scanner, as some preliminary results suggest [11], or even by interposing the aforementioned foils between each pair of RPC detectors. However, both solutions require more detailed studies. Nevertheless, and according to the results of Sect. 4.3, the increased SF is not expected to degrade the SR. Moreover, the analysis of the the distribution of the distances of the LORs to the annihilation points, suggest that scatter contributes to a long-range diffuse background $[11,10]$ that does not compromise the $\mathrm{SR}$, being also expected that it does not compromise the quality of the reconstructed image.

For completeness, it is worth to mention that the best results concerning the SF were, as expected, obtained for the coincidences processed with the STW sorter and the rejection of LORs made by GTOFR [10, 11]. However, the SF obtained with the MTW coincidence sorter and the GTOFR method was found to be increased only by $\approx 3 \%[10,11]$, being this increase due to long range scatter, which is not expected to compromise image quality.

\subsection{Noise equivalent count rate}

Figure 9 depicts the results obtained for the expected NECR (top plots) of the proposed RPC TOF-PET scanner, by performing the coincidences with the MTW coincidence sorter, followed by the rejection of LORs performed with the GTOFR method, for both simulated phantoms (the standard $700 \mathrm{~mm}$ long NEMA NU22001 [13], and the axially extended one, with an axial length of $1800 \mathrm{~mm}$ ). The plots also depict the experimental NECR curve reported by Surti et al. [21] for the Philips Gemini TF scanner, obtained with the standard $700 \mathrm{~mm}$ long NEMA NU2-2001 [13] phantom. The bottom plots, depict the expected gain in NECR of the proposed RPC TOF-PET scanner relative to that reported by Surti et al. [21] for the Philips Gemini TF scanner ob- 


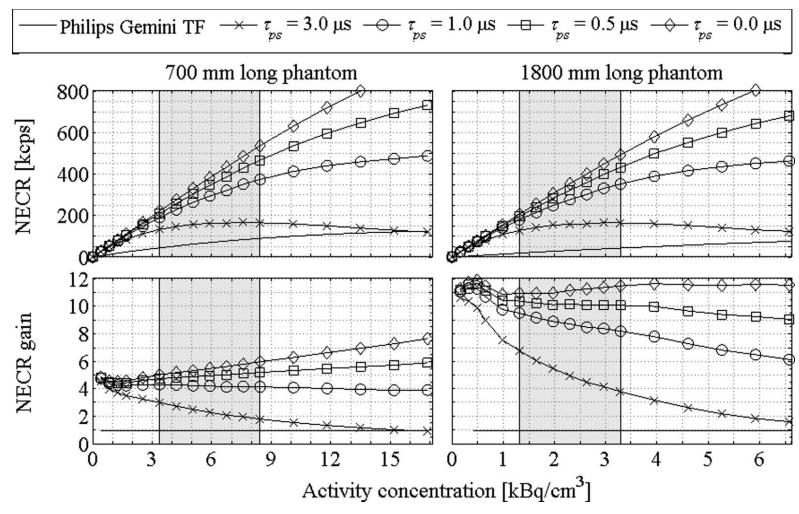

Fig. 9. NECR curves (top plots) obtained for both phantoms and all values of the $\tau_{\mathrm{ps}}$ dead time for position signals, for the MTW coincidence sorter followed by the removal of LORs using the GTOFR method [10, 11], along with experimental data reported by Surti et al. [21] for the Philips Gemini TF scanner obtained with the standard $700 \mathrm{~mm}$ long NEMA NU2-2001 [13] phantom. Also presented are the expected gains in NECR of the RPC TOF-PET scanner for all values of the $\tau_{\mathrm{ps}}$ dead time for position signals relative to the experimental data reported by Surti et al. [21] for the Philips Gemini TF scanner obtained with the standard $700 \mathrm{~mm}$ long NEMA NU2-2001 [13] phantom. Greyed portions correspond to total phantom activities ranging from $74 \mathrm{MBq}$ $(2 \mathrm{mCi})$ to $185 \mathrm{MBq}(5 \mathrm{mCi})$.

tained with the standard $700 \mathrm{~mm}$ NEMA NU2-2001 [13] phantom. Greyed portions correspond to total phantom activities ranging from $74 \mathrm{MBq}(2 \mathrm{mCi})$ to $185 \mathrm{MBq}$ $(5 \mathrm{mCi})$.

For the standard NEMA NU2-2001 [13] phantom, a peak NECR of $167 \mathrm{kcps}$ at $7.6 \mathrm{kBq} / \mathrm{cm}^{3}$ $\left(\approx 0.21 \mu \mathrm{Ci} / \mathrm{cm}^{3}\right)$, was obtained for the present experimental value of $\tau_{\mathrm{ps}}=3.0 \mu \mathrm{s}[10,11]$, which is $\approx 2.0$ times that reported by Surti et al. [21] for the Philips Gemini TF scanner with the same phantom at the same activity concentration. For the remaining values of the $\tau_{\mathrm{ps}}$ dead time for position signals, no peak value for the NECR was found for the activity concentrations employed in the simulations, the NECR increasing with the activity concentration. In particular, for a value of $\tau_{\mathrm{ps}}=1.0 \mu \mathrm{s}$ (seemingly achievable with dedicated efforts in speeding up the electronics and improving the SNR - signal to noise ratio), the NECR increased up to $485 \mathrm{kcps}$ at $16.8 \mathrm{kBq} / \mathrm{cm}^{3}\left(\approx 0.45 \mu \mathrm{Ci} / \mathrm{cm}^{3}\right)$, having been of $349 \mathrm{kcps}$ at $7.6 \mathrm{kBq} / \mathrm{cm}^{3}\left(\approx 0.21 \mu \mathrm{Ci} / \mathrm{cm}^{3}\right)[10,11]$. This represents a 4.2 gain in NECR relative to that reported by Surti et al. [21] for the Philips Gemini TF scanner with the same phantom and activity concentration.

For the $1800 \mathrm{~mm}$ axially extended phantom, and plotting the NECR against the total activity in the phantom, similar curves to those presented for the standard 700 mm length NEMA NU2-2001 [13] phantom, were obtained, although with a slight decrease of the NECR due to the decreasing solid angle coverage near the scanner ends [10, 11]. However, for the same total activity in the phantom there is $\mathrm{a} \approx 0.39(=700 / 1800)$ reduction in activity concentrations, which means that the peak NECR for the value of $\tau_{\mathrm{ps}}=3.0 \mu \mathrm{s}$, occurred for an activity concentration of $3 \mathrm{kBq} / \mathrm{cm}^{3}\left(\approx 0.08 \mu \mathrm{Ci} / \mathrm{cm}^{3}\right)$, the NECR having been of $164 \mathrm{kcps}[10,11]$, representing a gain of 5.5 relative to that reported by Surti et al. [21] for the Philips Gemini TF scanner with the strict NEMA NU2-2001 [13] phantom at the same activity concentration. By decreasing the $\tau_{\mathrm{ps}}$ dead time for position signals to a seemingly achievable value of $1.0 \mu \mathrm{s}$, the gain in the NECR increased to $8.3[10,11]$, relative to that reported by Surti et al. [21] for the Philips Gemini TF scanner and the strict $700 \mathrm{~mm}$ long NEMA NU2-2001 [13], for the same activity concentration.

\section{Conclusions}

Simulations suggest that the absolute 3D true sensitivity of an RPC TOF-PET scanner with an AFOV of $2400 \mathrm{~mm}$, and 120 gas amplification gaps in the radial direction, measured according to the 1994 and 2001 versions of the NEMA NU2 standards [12, 13], is expected to be higher than those presented by crystal-based PET scanners, by a factor of at least 4.1 for strict NEMA NU2 $[12,13]$ phantoms, if no gain factor due to TOF information $[17,18]$ is to be considered, the increase being higher for axially extended phantoms and/or if a gain factor due to TOF information $[17,18]$ is to be considered [6, 9, 11]. For an RPC TOF-PET scanner with more amplification gaps, the gain in sensitivity relative to crystal-based PET scanners is expected to be higher. However, the simulations of Ref. [6] and Ref. [9], and revised in the current paper, did not account for scatter in the detectors, and, as such, further investigation with the complete geometry of the proposed RPC TOF-PET scanner must be performed.

The simulations also suggest that the expected SR of the proposed RPC TOF-PET scanner, is expected to be of $\approx 2.3 \mathrm{~mm}$ FWHM, and of $\approx 4.9 \mathrm{~mm}$ FWTM, including the detector readout (considering $2 \mathrm{~mm}$ pitch strips and a pitch of $3.4 \mathrm{~mm}$ for the DOI), and photon acollinearity effects, but excluding the positron range $[10,11]$. These values are about twice as better than those reported for three commercially available state-of-the-art PET scanner with TOF information (the Philips Gemini TF [21], the GE Discovery 690 [22, 23], and the Siemens Biograph mCT [24]). The effect of photon acollinearity on the SR was found to be much lower than that generally reported in the literature [25]. This was also shown by reconstructing coincidence data affected by a $0.58^{\circ}$ FWHM Gaussian distribution in the direction of one photon relative to the direction of the other, obtained mathematically for an ideal unsegmented cylindrical PET scanner with a diameter of $1000 \mathrm{~mm}$ and an AFOV of $2400 \mathrm{~mm}$, indicating that the effect of acollinearity in the SR generally assumed in the literature [25] may be overestimated. These results deserve further research, not only by simulation, but also experimentally, by assessing the effect of photon acollinearity in the SR with detectors with higher 
intrinsic SR, as those currently employed in a pre-clinical RPC PET system.

An efficient trigger for the RPC TOF-PET scanner, based on a MTW coincidence sorter followed by rejection of those LORs for which the direct TOF reconstructed point falls outside a tight region surrounding the phantom, was proposed, which is able to efficiently reduce the random CRs by more than $70 \%$, holding the best results for the NECR $[11,10]$. However, the effect of such trigger in the SR that can be obtained for total activities in the FOV in the range of those that are expected to be present in clinical practice, and in the quality of the final reconstructed image, still needs to be assessed.

With the aforementioned trigger, the average SF obtained for the RPC TOF-PET scanner with the $700 \mathrm{~mm}$ long NEMA NU2-2001 [13] phantom, was of $\approx 52 \%$, and of $\approx 54 \%$ for the $1800 \mathrm{~mm}$ axially extended phantom. These values are much higher than those reported for three commercially available state-of-the-art PET scanner with TOF information (the Philips Gemini TF [21], the GE Discovery 690 [22, 23], and the Siemens Biograph mCT [24]). However, it was found that scatter contributes to a long-range diffuse background that does not compromise the SR $[10,11]$, being also expected that it does not compromise the quality of the reconstructed image. However, to clarify on the effect of the influence of the scatter on the quality of the final reconstructed image, the image quality must still be accessed by simulation. Nevertheless, further simulations must be performed to access the possibility of decreasing the SF by adding foils of appropriate material and thickness to the surface of the scanner detection heads, as well as interposing the aforementioned foils between consecutive RPC detectors.

As to the NECR obtained for the RPC TOF-PET scanner with the $700 \mathrm{~mm}$ long NEMA NU2-2001 [13] phantom, and for a value of $3.0 \mu \mathrm{s}$ for the dead time for position signals, was found to rise from $133 \mathrm{kcps}$ to a peak value of $167 \mathrm{kcps}$ decreasing then to $164 \mathrm{kcps}$, for activity concentrations of, respectively, $3.4 \mathrm{kBq} / \mathrm{cm}^{3}$, $7.6 \mathrm{kBq} / \mathrm{cm}^{3}$, and $8.4 \mathrm{kBq} / \mathrm{cm}^{3}[10,11]$. These values correspond to NECR increases of, respectively, 2.9, 1.8, and 1.7 relative to experimental values reported by Surti et al. [21] for the Philips Gemini TF scanner, obtained with the same phantom at the same activity concentrations $[10,11]$. For a $1.0 \mu \mathrm{s}$ dead time for the position signals (seemingly achievable with dedicated efforts to improve the SNR and to speed up electronics) no peak NECR was found, the NECR at the aforementioned activity concentrations being, respectively, $189 \mathrm{kcps}, 349 \mathrm{kcps}$, and $372 \mathrm{kcps}$, representing an increase of, respectively, $4.1,3.7$, and 3.6 relative to experimental values reported by Surti et al. [21] for the Philips Gemini TF scanner with the same phantom at the same activity concentrations $[10,11]$. For the $1800 \mathrm{~mm}$ axially extended phantom, activity concentrations comprised between $1.3 \mathrm{kBq} / \mathrm{cm}^{3}$ to $3.3 \mathrm{kBq} / \mathrm{cm}^{3}$, corresponding to total activities in the phantom of, respectively, $74 \mathrm{MBq}$
$(2 \mathrm{mCi})$ and $185 \mathrm{MBq}(5 \mathrm{mCi})$, and considering a value of $3.0 \mu \mathrm{s}$ for the dead time for position signals, the NECR values of the proposed RPC TOF-PET were found to be, respectively, 6.8 and 3.8 times higher than experimental values reported by Surti et al. [21] for the Philips Gemini TF scanner obtained with the standard $700 \mathrm{~mm}$ long NEMA NU2-2001 [13] phantom, for the same activity concentrations $[10,11]$. For a seemingly achievable reduction in the dead time for position signals to $1.0 \mu \mathrm{s}$, and for the aforementioned activity concentrations, the gains in the NECR values obtained for the RPC TOFPET were, respectively, 9.5 and 8.1 higher than those reported by Surti et al. [21] for the Philips Gemini TF scanner [10, 11].

As a final conclusion, although RPC detectors seem an unlikely candidate for a successful detector for PET applications, mainly due to the lack of energy resolution and low quantum efficiency, the results obtained so far, are very promising in what concerns the viability of a large AFOV RPC TOF-PET scanner, leading the authors to continue the research efforts toward a full functional prototype.

\section{Acknowledgments}

The authors are grateful to F. Caramelo, from the Faculty of Medicine of the University of Coimbra, for his valuable discussions, and to J. Silva and M. Oliveira, from the Laboratory of Instrumentation and Experimental Particle Physics (LIP), for their technical support.

The authors gratefully acknowledge the Laboratory of Advanced Computing of the University of Coimbra, for the generous computation time provided.

This work was financed by the FCT and FEDER under Contracts POCI/FP/63411/2005, POCI/SAUOBS $/ 61642 / 2004$, by the EU, FEDER, POCI, QREN, COMPETE and FCT under Contracts CERN-FP-835242008 and PTDC/SAU-BEB/104630/2008, and by the Instituto de Investigação Interdisciplinar of the University of Coimbra.

The work of M. Couceiro was partially supported by the FCT grants SFRH/BD/42217/2007 and SFRH/PROTEC/49822/2009. The work of P. Crespo was supported by the FCT grant SFRH/BPD/39223/ 2007 .

\section{References}

[1] A. Blanco, V. Chepel, R. Ferreira-Marques, P. Fonte, M.I. Lopes, V. Peskov, A.R. Policarpo, Nucl. Instrum. Methods Phys. Res. A 508, 88 (2003).

[2] J.E. Bateman, J.F. Connolly, R. Stephenson, A.C. Flesher, Nucl. Instrum. Methods 176, 83 (1980).

[3] A. Blanco, P. Fonte, L. Lopes, P. Martins, J. Michel, M. Palka, M. Kajetanowicz, G. Korcyl, M. Traxler, R. Marques, J. Instr. 7, P11012 (2012).

[4] A. Blanco, N. Carolino, C.M.B.A. Correia, L. Fazendeiro, N.C. Ferreira, M.F.F. Marques, R.F. Marques, P. Fonte, C. Gil, M.P. Macedo, IEEE Trans. Nucl. Sci. 53, 2489 (2006). 
[5] A. Blanco, M. Couceiro, P. Crespo, N.C. Ferreira, R. Ferreira Marques, P. Fonte, L. Lopes, J.A. Neves, Nucl. Instrum. Methods Phys. Res. A 602, 780 (2009).

[6] M. Couceiro, A. Blanco, N.C. Ferreira, R. Ferreira Marques, P. Fonte, L. Lopes, Nucl. Instrum. Methods Phys. Res. A 580, 915 (2007).

[7] M. Couceiro, N.C. Ferreira, P. Fonte, Nucl. Instrum. Methods Phys. Res. A 580, 485 (2007).

[8] M. Couceiro, P. Crespo, L. Mendes, N. Ferreira, R. Ferreira Marques, P. Fonte, Nucl. Instrum. Methods Phys. Res. A 661, S156 (2012).

[9] P. Crespo, J. Reis, M. Couceiro, A. Blanco, N.C. Ferreira, R. Ferreira Marques, P. Martins, P. Fonte, IEEE Trans. Nucl. Sci. 59, 520 (2012).

[10] M. Couceiro, P. Crespo, R.F. Marques, P. Fonte, IEEE Trans. Nucl. Sci. 61, 1153 (2014).

[11] M. Couceiro, Ph.D. Thesis, University of Coimbra, Coimbra 2014.

[12] National Electrical Manufacturers Association, NEMA Standards Publication NU 2-1994 (1994).

[13] National Electrical Manufacturers Association, NEMA Standards Publication NU 2-2001 (2001).

[14] S. Agostinelli, J. Allison, K. Amako, J. Apostolakis, H. Araujo, P. Arce, M. Asai, D. Axen, S. Banerjee, G. Barrand, F. Behner, L. Bellagamba, J. Boudreau, L. Broglia, A. Brunengo, H. Burkhardt, S. Chauvie, J. Chuma, R. Chytracek, G. Cooperman, G. Cosmo, P. Degtyarenko, A. Dell'Acqua, G. Depaola, D. Dietrich, R. Enami, A. Feliciello, C. Ferguson, H. Fesefeldt, G. Folger, F. Foppiano, A. Forti, S. Garelli, S. Giani, R. Giannitrapani, D. Gibin, J.J. Gómez Cadenas, I. González, G. Gracia Abril, G. Greeniaus, W. Greiner, V. Grichine, A. Grossheim, S. Guatelli, P. Gumplinger, R. Hamatsu, K. Hashimoto, H. Hasui, A. Heikkinen, A. Howard, Nucl. Instrum. Methods Phys. Res. A 506, 250 (2003).

[15] J. Allison, K. Amako, J. Apostolakis, H. Araujo, P.A. Dubois, M. Asai, G. Barrand, R. Capra, S. Chauvie, R. Chytracek, G.A.P. Cirrone, G. Cooperman, G. Cosmo, G. Cuttone, G.G. Daquino, M. Donszelmann, M. Dressel, G. Folger, F. Foppiano, J. Generowicz, V. Grichine, S. Guatelli, P. Gumplinger, A. Heikkinen, I. Hrivnacova, A. Howard, S. Incerti, V. Ivanchenko, T. Johnson, F. Jones, T. Koi, R. Kokoulin, M. Kossov, H. Kurashige, V. Lara, S. Larsson, F. Lei, O. Link, F. Longo, M. Maire, A. Mantero, B. Mascialino, I. McLaren, P.M. Lorenzo, K. Minamimoto, K. Murakami, P. Nieminen, L. Pandola, S. Parlati, L. Peralta, J. Perl, A. Pfeiffer, M.G. Pia, A. Ribon, P. Rodrigues, G. Russo, S. Sadilov, G. Santin, T. Sasaki, D. Smith, N. Starkov, S. Tanaka, E. Tcherniaev, B. Tome, A. Trindade, P. Truscott, L. Urban, M. Verderi, A. Walkden, J.P. Wellisch, D.C. Williams, D. Wright, H. Yoshida, IEEE Trans. Nucl. Sci. 53, 270 (2003).
[16] S. Jan, G. Santin, D. Strul, S. Staelens, K. Assié, D. Autret, S. Avner, R. Barbier, M. Bardiés, P.M. Bloomfield, D. Brasse, V. Breton, P. Bruyndonckx, I. Buvat, A.F. Chatziioannou, Y. Choi, Y.H. Chung, C. Comtat, D. Donnarieix, L. Ferrer, S.J. Glick, C.J. Groiselle, D. Guez, P.-F. Honore, S. Kerhoas-Cavata, A.S. Kirov, V. Kohli, M. Koole, M. Krieguer, D.J. van der Laan, F. Lamare, G. Largeron, C. Lartizien, D. Lazaro, M.C. Maas, L. Maigne, F. Mayet, F. Melot, C. Merheb, E. Pennacchio, J. Perez, U. Pietrzyk, F.R. Rannou, M. Rey, D.R. Schaart, C.R. Schmidtlein, L. Simon, T.Y. Song, J.-M. Vieira, D. Visvikis, R. Van de Walle, E. Wieërs, C. Morel, Phys. Med. Biol. 49, 4543 (2004).

[17] W.W. Moses, IEEE Trans. Nucl. Sci. 50, 1325 (2003).

[18] M. Conti, Phys. Med. 25, 1 (2009).

[19] T.K. Lewellen, S.G. Kohlmyer, R.S. Miyaoka, C.W. Stearns, IEEE Trans. Nucl. Sci. 42, 1051 (1995).

[20] S.G. Kohlmyer, C.W. Stearns, P.E. Kinahan, T.K. Lewellen, in: Conf. Rec. 2002 IEEE Nuclear Science Symposium and Medical Imaging Conference, Vol. 2, Nuclear Science Symposium Conference Record, 2002 IEEE, Norfolk 2002, p. 890.

[21] S. Surti, A. Kuhn, M.E. Werner, A.E. Perkins, J. Kolthammer, J.S. Karp, J. Nucl. Med. 43, 471 (2007).

[22] B. Kemp, J. Williams, R. Ruter, V. Lowe, B. Mullan, J. Nucl. Med. 50, 1546 (2009).

[23] V. Bettinardi, L. Presotto, E. Rapisarda, M. Picchio, L. Gianolli, M.C. Gilardi, Med. Phys. 38, 5394 (2011).

[24] B.W. Jakoby, Y. Bercier, M. Conti, M.E. Casey, B. Bendriem, D.W. Townsend, Phys. Med. Biol. 56, 2375 (2011).

[25] W.W. Moses, Nucl. Instrum. Methods Phys. Res. A 648, S236 (2011).

[26] S. Vandenberghe, J. Karp, Nucl. Instrum. Methods Phys. Res. A 569, 421 (2006).

[27] M.E. Daube-Witherspoon, J.S. Karp, M.E. Casey, F.P. DiFilippo, H. Hines, G. Muehllehner, V. Simcic, C.W. Stearns, L.E. Adam, S. Kohlmyer, V. Sossi, J. Nucl. Med. 43, 1398 (2002).

[28] P. Martins, A. Blanco, P. Crespo, M.F. Ferreira Marques, R. Ferreira Marques, P.M. Gordo, M. Kajetanowicz, G. Korcyl, L. Lopes, J. Michel, M. Palka, M. Traxler, P. Fonte, J. Inst. 9, C10012 (2014). 